\title{
A!
}

This is an electronic reprint of the original article.

This reprint may differ from the original in pagination and typographic detail.

Piirainen, Mari A.; de Ruijter, Jorg C.; Koskela, Essi V.; Frey, Alexander D.

\section{Glycoengineering of yeasts from the perspective of glycosylation efficiency}

Published in:

NEW BIOTECHNOLOGY

DOI:

10.1016/j.nbt.2014.03.001

Published: 25/12/2014

Document Version

Peer reviewed version

Published under the following license:

CC BY-NC-ND

Please cite the original version:

Piirainen, M. A., de Ruijter, J. C., Koskela, E. V., \& Frey, A. D. (2014). Glycoengineering of yeasts from the perspective of glycosylation efficiency. NEW BIOTECHNOLOGY, 31(6), 532-537.

https://doi.org/10.1016/j.nbt.2014.03.001

This material is protected by copyright and other intellectual property rights, and duplication or sale of all or part of any of the repository collections is not permitted, except that material may be duplicated by you for your research use or educational purposes in electronic or print form. You must obtain permission for any other use. Electronic or print copies may not be offered, whether for sale or otherwise to anyone who is not an authorised user. 


\section{Glycoengineering of yeasts from the perspective of glycosylation efficiency}

Running title: Not only the glycoform matters

Mari A. Piirainen, Jorg C. de Ruijter, Essi V. Koskela, Alexander D. Frey

Department of Biotechnology and Chemical Technology, Aalto University, Finland

Corresponding author:

Alexander D. Frey

P.O. Box PL 16100

FI-00076 Aalto

alexander.frey@aalto.fi

+358504116506

Keywords: N-glycosylation, lipid-linked oligosaccharide biosynthesis, protozoan oligosaccharyltransferase, hypoglycosylation, glycoengineering, yeast 


\begin{abstract}
$\mathrm{N}$-linked glycosylation of proteins is one of the most common posttranslational modifications. Nglycan structures as well as $\mathrm{N}$-glycosylation efficiency are critical parameters in the production of $\mathrm{N}$-glycosylated proteins. Yeast cells can be seen as an attractive production host for therapeutic glycoproteins and pioneering work of glycoengineering was performed in Pichia pastoris, realizing yeast strains capable of producing defined, human-type N-glycans.

Most strategies used for glycoengineering rely on the modification of the lipid-linked oligosaccharide biosynthesis for the generation of the substrate for Golgi-localized glycosyltransferases. However, modifications in the lipid-linked oligosaccharide biosynthesis often result in the accumulation of intermediate structures and cause hypoglycosylation of client proteins. In order to ensure complete $\mathrm{N}$-glycosylation, the flow of lipid-linked oligosaccharide through the biosynthetic pathway and the transfer of the oligosaccharide from the donor lipid onto the protein have to be optimized. A promising tool to improve site occupancy is the expression of protozoan oligosaccharyltransferases, which possess altered specificities for the oligosaccharide and also for the protein acceptor site. Furthermore, flipping of the lipid-linked oligosaccharide into the ER lumen can be improved by overexpression of an artificial flippase. Improving the glycosylation efficiency ensures that not only homogeneous N-glycan structures are generated, but also client proteins are fully glycosylated.
\end{abstract}

\title{
Introduction
}

$\mathrm{N}$-linked glycosylation of proteins (N-glycosylation) is one of the most common posttranslational modifications. N-glycosylation is species-specific and typically very heterogeneous, and it is important for protein folding, solubility, stability, and functions such as in vivo activity, serum halflife and immunogenicity [1,2]. Currently, most therapeutic glycoproteins are produced in mammalian cell lines, such as Chinese hamster ovary cells and mouse myeloma cells.

$\mathrm{N}$-glycans play a well-known role in the performance of therapeutic proteins. Structure of the individual glycans as well as the number of the N-glycan on the proteins can have a significant effect on the therapeutic performance [3]. Therefore, glycoengineering is a viable approach in order to improve the functionality of proteins. A well-known example is the role of the fucose residue attached to the core of the $\mathrm{N}$-glycan on the binding of the antibody to the cognate $\mathrm{F}_{\mathrm{c}}$ receptor and the subsequent activation of the antigen-dependent cellular toxicity. Expression of the antibody in a glycoengineered cell line yielded a non-fucosylated version of the antibody which displayed an increased affinity of the IgG to the $\mathrm{F}_{\mathrm{c}}$ receptor, coinciding with an increased antigen-dependent 
cellular cytotoxicity [4]. Core fucosylation can modulate the affinity of the IgG with the cognate $\mathrm{F}_{\mathrm{C}}$ receptor.

Besides the glycoform, also the number of N-glycans attached to the protein affects protein function and therefore its therapeutic performance. Human erythropoietin (hEPO) is a small glycoprotein carrying three N-glycosylation sites. An aglycosylated but biologically active form of hEPO can be produced in E. coli, but the absence of the N-glycans strongly reduced the in vivo half-life. In contrast, engineering of an EPO variant carrying two additional N-glycan chains (Darbepoietin alfa) increased in vivo half-life and activity [5].

Clearly, the structures and number of N-glycan are powerful modulators of the activity of therapeutic proteins but both features can be adversely affected by shortcomings during production or by the choice of the production system. N-glycosylation does not occur at every potential glycosylation site and variations in the site occupancy are a function of the glycosylation site sequence and its structural context, the concentration and structure of the lipid-linked oligosaccharide (LLO), and enzyme kinetics. Whereas the glycosylation site of a given protein is defined, and in the case of therapeutic proteins the structural context of the N-glycosylation site cannot be altered, factors affecting the concentration and structures of LLO and the kinetics and specificities of the oligosaccharyltransferase (OST) can have a negative impact on the site occupancy. This review summarizes the current strategies for glycoengineering of yeast expression systems, and their impact on glycosylation efficiency.

\section{Glycoengineering in yeasts}

Yeast cells have been commonly used in biotechnology to produce a wide range of industrial enzymes and metabolites, but also for the production of therapeutic proteins, such as insulin and Hepatitis A vaccine [6]. Yeast cells can be seen as an attractive production host for therapeutic glycoproteins, since yeast cells have many advantages over mammalian cells. The productivities obtained with yeast cells can be higher, since yeast cells have a faster growth rate and high protein yields can be obtained. Due to the more rigid cell wall, yeast cells are also less fragile than mammalian cells and thus more resistant to shear stress. Yeast can also grow in chemically defined, serum-free media, which lowers the costs of cultivation and removes the concern of contaminations by viruses, endotoxins and prions. For these reasons, production of glycoproteins with yeast cells would be easier and cheaper compared to mammalian production systems, especially in large-scale production.

Being a eukaryote, yeast is capable of performing posttranslational modifications, including Nglycosylation. However, the N-glycan structures in yeast glycoproteins differ significantly from 
human N-glycans. Yeast N-glycans are highly mannosylated structures. In contrast, the N-glycan structures in mammalian cells are more complex and contain monosaccharides not commonly present in yeast N-glycans, such as fucose, galactose and sialic acid [2].

In order to produce human-like glycoproteins with yeast, the N-glycosylation pathways of yeast cells need to be modified. Research on this subject has been pioneered in Pichia pastoris [7,8]. However, very recently Parsaie Nasab et al. (2013) were able to produce a monoclonal antibody that contained a human-like N-glycan structure in a glycoengineered Saccharomyces cerevisiae strain [9].

The glycoengineering of yeasts has been extensively reviewed, with a strong focus on the steps taking place in the Golgi apparatus [7,8]. The architecture of Golgi-localized glycosyltransferases and mannosidases is highly conserved between species and consists of an N-terminal transmembrane domain (TMD) with an extension into the cytoplasm of different length, a stem domain and a C-terminal catalytic domain. In order to target vertebrate glycosyltransferases and mannosidases in the Golgi apparatus of yeast, fusions of TMD and stem domains of yeast mannosyltransferases with catalytic domains of vertebrate glycosyltransferases and mannosidases have been created, selecting for each enzyme activity the optimal TMD and stem domain [10]. Expression of these hybrid mannosidases and glycosyltransferases enabled the generation of sialylated, complex-type N-glycans.

Five different strategies have been pursued so far for the glycoengineering of yeasts and fungi, which will be briefly summarized. The approaches differ in the way the substrate for Golgilocalized N-acetyglucosaminyltransferase I (GnTI) and N-acetyglucosaminyltransferase II (GnTII) are generated. Common to mammals and yeast is the LLO biosynthesis pathway culminating in the generation of the Glc $\mathrm{Man}_{9} \mathrm{GlcNAc}_{2}$ structure, which is transferred onto protein by the OST complex. During the protein folding process ER glucosidase I and II and ER mannosidase trim the $\mathrm{N}$-glycan to its final Man ${ }_{8} \mathrm{GlcNac}_{2}$ structure, which leaves the ER (Figure 1A). After this step Nglycosylation pathways of mammals and yeast diverge. In mammals the $\alpha-1,2$ linked mannose residues of the N-glycan are trimmed off by mannosidase I (ManI) creating the substrate $\left(\mathrm{Man}_{5} \mathrm{GlCNac}_{2}\right.$ ) for GnTI. After the transfer of the first GlcNAc by GnTI, mannosidase II (ManII) cleaves two additional mannose residues creating GlcNAcMan ${ }_{3} \mathrm{GlcNAc}_{2}$, which serves as substrate for GnTII resulting in the most simple complex N-glycan GlcNAc $2 \mathrm{Man}_{3} \mathrm{GlcNAc}_{2}$.

In the first successful approach to humanize N-glycosylation in yeast, the mammalian $\mathrm{N}$ glycosylation pathway was reproduced in yeast introducing mannosidase activities (ManI and ManII) and glycosyltransferases (GnTI and GnTII) into the Golgi apparatus, which enabled the generation of complex-type N-glycans (Figure 1A) [11]. 
In vitro studies with human GnTI have shown that this enzyme is tolerating shorter N-glycans as acceptor, indeed GnTI is active on $\mathrm{Man}_{3} \mathrm{GlcNAc}_{2}$ [12]. Therefore, it seemed plausible that the glycosylation pathway in yeast might be simplified by providing a tailored N-glycan as substrate for GnTI. Several alternative routes have been described for generation of the substrate for GnTI and GnTII (Figure 1). These modified routes are based on the modification of the LLO biosynthesis pathway in yeast, the introduction of an $\alpha 1,2$ mannosidase ( $\alpha 1,2-M a n)$ activity into the ER lumen or a combination of both of these two approaches. Depending on the approach a Man ${ }_{3} \mathrm{GlcNAc}_{2}$, the biosynthetic Man ${ }_{5} \mathrm{GlcNAc}_{2}$ structure lacking the B- and C-branch or a trimmed Man ${ }_{5} \mathrm{GlCNAc}_{2}$ being devoid of all $\alpha 1,2-$ mannose residues are generated. The latter two structures still require the activity of Golgi-localized ManI or ManII activities, respectively.

Expression of a fungal $\alpha 1,2-$ Man in the ER lumen trims all $\alpha 1,2$ mannose residues creating a Man $_{5}$ GlcNAc $_{2}$ structure, which can serve as substrate for GnTI (Figure 1B). Deletion of ALG3 gene creates the biosynthetic Man ${ }_{5}$ GlcNAc$_{2}$ LLO lacking the B- and C-branch. Alg3 is an $\alpha 1,3-$ mannosyltransferase, which initiates the elongation of the LLO in the ER lumen. Its activity exerts a high degree of control on LLO processing in the ER. The absence of this $\alpha 1,3$-linked mannose terminates all further modifications of the LLO and this structure is transferred by the OST complex with a strongly reduced efficiency [13]. In order to create the substrate for GnTI and GnTII only a

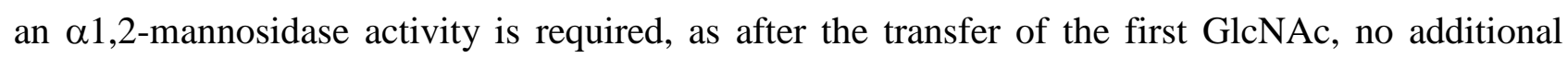
trimming of mannoses is required and the GlcNAcMan ${ }_{3} \mathrm{GlcNAc}_{2}$ can directly serve as substrate for GnTII. Two strategies for the localization of the $\alpha 1,2$ mannosidase activity have been pursued. Either ManI activity is destined to the early Golgi apparatus [10] or a fungal $\alpha 1,2$ mannosidase ( $\alpha 1,2-\mathrm{Man})$ is targeted into the ER and the $\mathrm{Man}_{3} \mathrm{GlcNAc}_{2}$ structure is already generated there [14] (Figure 1C and D).

The most recent strategy is based on the idea that a LLO can be tailored, which without additional trimming can serve as substrate for GnTI and GnTII [9]. Deletion of ALG11 and ALG3 genes in the LLO biosynthesis pathway leads to the formation of $\mathrm{Man}_{3} \mathrm{GlcNAc}_{2}$. Alg11 is catalysing the last step on the cytoplasmic side of the ER lumen adding two $\alpha 1$,2-mannoses, which form the A-branch of the glycan. Consequently, deletion of the $A L G 11$ gene prevents formation of the A-branch; in combination with ALG3 deletion none of the branches of the glycan are formed creating the $\mathrm{Man}_{3} \mathrm{GlcNAc}_{2}$ structure. This structure, when present on a protein serves as a substrate for GnTI and GnTII (Figure 1E).

\section{Modification of the LLO-biosynthesis and its effects on N-glycosylation efficiency}


As discussed above most of the strategies for generation of the substrates for GnTI and GnTII rely on the modification of the LLO biosynthesis pathway. In the next paragraphs we would like to discuss the impact of manipulation of the LLO-biosynthesis on the N-glycosylation efficiency. LLO biosynthesis is initiated at the cytoplasmic side of the ER, where a dolichol-phosphate (Dol-P) serves as an acceptor of GlcNAc-phosphate forming Dol-PP-GlcNAc in a reaction catalysed by Alg7. The second GlcNAc residue is added by Alg13/Alg14 complex. The first mannose is added by Alg1, after which an $\alpha 1$,3-linked and an $\alpha 1$,6-linked mannose are added by Alg2, creating the Man $_{3} G_{\text {GlNAc2 }}$ structure. Before flipped into the ER lumen two $\alpha 1$,2-linked mannoses are added to the $\alpha 1$,3-linked mannose creating the biosynthetic Man ${ }_{5}$ GlcNAc$_{2}$ structure. This structure is flipped into the ER lumen, originally thought by the RFT1 protein [15]. Alg3 initiates the lumenal steps of the LLO biosynthesis adding an $\alpha-1,3$ linked mannose. An $\alpha 1,2-$ linked mannose is added by Alg9 finalizing the B-branch, before the C-branch is initiated by addition of $\alpha-1,6$ linked mannose by Alg12. This structure is capped by an $\alpha-1,2$ linked mannose added by Alg9. After generation of the Man $_{9}$ GlcNAc$_{2}$ structure, three glucose units are added to the A-branch (Figure 2). The glucose residues are the key-signature for the substrate recognition by the OST complex. Deficiencies in the LLO assembly result in the accumulation of intermediate structures and cause hypoglycosylation, a fact which is very well documented in S. cerevisiae.

Deletion of the ALG3 gene in S. cerevisiae has detrimental effects on LLO biosynthesis in the ER lumen, as the deletion aborts the lumenal extension of the growing LLO, generating mainly Man $_{5}$ GlcNac $_{2}$ structures [13]. As stated above, the capping glucose residues are key determinants for the recognition of the LLO by the OST complex, therefore, N-glycosylation efficiency is strongly reduced in an alg3 mutant leading to severe hypoglycosylation of proteins, as has been shown for several endogenous glycoproteins (CPY, Wbp1p, Gas1p)[13]. Recently, the ALG3 gene from $P$. pastoris has been characterized, however, the effect of its deletion on glycosylation efficiency has not been analyzed [16]. The glycoengineering strategy based on the ALG3 deletion was also utilized in Yarrowia lipolytica, resulting in hypoglycosylation. In order to compensate for the reduced glycosylation efficiency, the ALG6 gene, encoding the glucosyltransferase adding the first glucose unit was overexpressed, which led to a partial shift from Man ${ }_{5} \mathrm{GlcNAc}_{2}$ in the $\Delta a \lg 3$ mutant to glucosylated forms of Man ${ }_{5} \mathrm{GlcNAc}_{2}$ structures in the ALG6 overexpressing strain [17]. As a result, hypoglycosylation of a client protein was attenuated. Overall, in the glycoengineering approaches based on the ALG3 deletion, the specificity of the OST complex for the LLO is the primary factor limiting glycosylation efficiency. 
When tailoring LLO biosynthesis in order to directly generate the substrate for Golgi-localized glycosyltransferases, the $A L G 3$ deletion is combined with the $A L G 11$ deletion. This strategy stops LLO synthesis already during the cytoplasmic steps creating the Man ${ }_{3} \mathrm{GlcNAc}_{2}$. Deletion of alg11 precludes the formation of the A-branch of the LLO, which leads to a very strong hypoglycosylation [18]. In addition to the reduced transfer efficiency, inefficient flipping of the LLO into the ER lumen contributes to the hypoglycosylation phenotype observed in the $\Delta a \lg 11$ strain. It had been reported previously that in a $\Delta a l g 11$ strain $\mathrm{Man}_{3} \mathrm{GlcNAc}_{2}$ accumulated on the cytoplasmic side of the ER membrane indicating that the flipping reaction is limiting. Upon overexpression of RFT1 a shift towards more mature LLO was observed, indicating that the flipping reaction can be improved [15]. The LLO flipped into the ER lumen are identical in the $\Delta a \lg 11$ and $\Delta a \lg 3 \Delta a \lg 11$ strains. Therefore, in order to realize an efficient glycosylation of client proteins in $\Delta$ alg3 $\Delta a \lg 11$ strain, both flipping and transfer have to be optimized.

\section{Improving N-glycosylation efficiency using protozoan oligosaccharyltransferases}

The OST complex in fungi and vertebrates is composed of 8 subunits, STT3 being the catalytically active subunit. In multi-subunit OST complex, some of the additional subunits fine-tune the $\mathrm{N}$ glycosylation process. Ost1 acts as a chaperon to promote glycosylation of selected protein clients. Ost3/6 exhibit oxidoreductase activity and bind specific polypeptides, both non-covalently and via transient mixed disulfide bonds. These subunits are thought to slow down the early stages of protein folding, thereby maintaining the polypeptide chain in a glycosylation-competent conformation. In addition to the catalytic subunit STT3, four additional subunits are essential (WBP1, OST1, OST2, SWP1) [1].

The complexity of the OST complex is reduced in Trichomonas, Entamoeba and Plasmodium where only four subunits are present. The most rigorous reduction in OST subunits is observed in Kinetoplastids and Giardia lamblia, which possess only the catalytic subunit STT3 and referred to as protozoan oligosaccharyltransferase (POT) [19]. However, the genomes of Leishmania spp and Trypanosomas spp comprise several genes encoding STT3 paralogous.

Furthermore, when analysing the genomes of Leishmania spp, and Trypanosomas spp, no homologues of glucosyltransferases (Alg6, Alg8 and Alg10) can be detected. Furthermore, the gene encoding Alg12 is absent in L. major, indicating that L. major produces a truncated LLO lacking the C-branch [20]. Therefore, it is plausible that POTs do not require the glucosyl capped A-branch for efficient transfer and might generally have an altered LLO specificity. Notably, dependent on the developmental cycle, LLO of varying length are formed. The organisms cope with this variation in the LLO structures, by expressing a distinct set of STT3 paralogous. T. brucei possess three 
STT3 paralogoues, TbSST3A being specific for Man ${ }_{5}$ GlcNAc $_{2}$, TbSTT3B being specific for $\mathrm{Man}_{9} \mathrm{GlcNAc}_{2}$ [21].

Several studies revealed that POT from L. major and T. brucei can replace the endogenous STT3 in S. cerevisiae and act independently from the other subunits of the OST complex [22,23]. Indeed, all of the essential subunits of the OST complex can be deleted in the presence of the single-subunit POTs. Furthermore, it was observed that POTs can transfer the glycan to other glycosylation sites than the one glycosylated by the endogenous OST complex. TbSTT3B and TbSTT3C when replacing yeast STT3, did have different activities towards some of the glycosylation sites, furthermore, sites which are normally not occupied were glycosylated by T. brucei STT3B and STT3C [21]. TbSTT3A was highly selective for acidic peptide acceptor sites, TbSTT3B has a broad specificity for the acceptor sites, whereas TbSTT3C preferentially glycosylated acidic peptide acceptors. When analyzing the site occupancy in yeast cells expressing different L. major STT3 paralogous, a similar (LmSTT3D) or reduced N-glycosylation efficiency (LmSTT3A and LmSTT3B) was observed [23]. Based on these observations, it is a fair assumption that POTs possess distinct specificities for the LLO and the glycosylation site and therefore represent an interesting tool for improving glycosylation efficiency.

POTs have been successfully utilized to increase $\mathrm{N}$-glycosylation efficiency in glycoengineered $P$. pastoris and S. cerevisiae. Glycoengineered P. pastoris produced only partially glycosylated antibodies, the glycosylation efficiency being 80\% [24]. Therefore, it has been evaluated whether the overexpression of POT would improve the glycosylation efficiency of heterologous client glycoproteins. The site occupancy of two antibodies (anti-Her2, anti-RSV) and rhGM-CSF was studied. Expression of LmSTT3D raised site occupancy of the two antibodies from 80 to $>99 \%$, but no improvement was seen when expressing one of the other L. major STT3 paralogous. In contrast, in the case of rhGM-CSF, which carries two N-glycosylation sites, only a partial attenuation of the hypoglycosylation could be observed upon coexpression of LmSTT3D, indicating that the structural context around the glycosylation sequon of the client protein itself is also an important determinant for the glycosylation efficiency.

In order to improve N-glycosylation efficiency in an $\Delta$ alg3 $\Delta$ alg11 yeast strain, we screened POTs isolated from Leishmania spp in yeast strains with altered LLO biosynthesis. A POT isolated from L. brasiliensis (LbSTT3_3) most significantly improved N-glycosylation efficiency in the target strain, but notably tolerated also a wide range of different LLO as substrate. As STT3 are integral membrane proteins, it proved to be essential to control expression levels, as to strong expression unnecessarily burdened cellular viability [9]. 
Unfortunately, no structural information of POTs is yet available. Therefore, the targeted modification of its properties such as LLO specificity or preference for N-glycosylation sequon is still not feasible.

\section{Improving $\mathbf{N}$-glycosylation efficiency using flippases}

Helenius et al. concluded that the ER membrane protein Rft1 is required directly for flipping of the LLO [15]. By replacing its native promoter with a galactose inducible promoter, Rft1 expression was suppressed by addition of glucose, and the Rft1 protein was depleted from cells. In these experiments Man ${ }_{5} \mathrm{GlCNAc}_{2}$ accumulated on the cytoplasmic side of the ER membrane and the impaired flipping was accompanied by hypoglycosylation in Rft1 depleted cells [15]. Although influencing the flipping, the assignment of Rft1 as the flippase was recently challenged by biochemical studies.

Using an $\Delta a \lg 11$ mutant, which prevents formation of the A-branch, but which after flipping of the LLO into the ER lumen is further elaborated to a Man ${ }_{7} \mathrm{GlcNAc}_{2}$ structure, flipping efficiency can be analyzed. In the $\Delta a l g 11$ mutant strain Man $_{3}$ GlcNAc2 $_{2}$ is accumulating, however upon overexpression of RFT1 a shift towards more mature LLO was observed, indicating that the flipping reaction can be improved [15].

Based on a genetic suppressor strain, we were able to identify a protein, which can act as an artificial flippase in yeast. Flc2* is a truncated form of the yeast Flc2 protein. FLC2 encodes a putative ER-localized FAD transporter. Flc2* is truncated after the first four transmembrane domains and its overexpression increased the flipping in $\Delta$ alg11 and $\Delta$ alg3 $\Delta$ alg11 mutants as judged from the attenuation of the hypoglycosylation phenotype in yeast [9]. Flc2* is a substrate unspecific flippase as it improved also the flipping of very short LLO such as Man ${ }_{1}$ GlcNAc$_{2}$. The mechanism, by which Flc2* is influencing the flipping remains elusive. By combining the Flc2* with LbSTT3_3 increased N-glycosylation efficiency to almost 100\% [9].

\section{Future perspectives: The effect of truncated LLOs on protein folding and cellular quality control systems}

Glycoengineering has generated valuable yeast strains, which can build almost completely homogeneous $\mathrm{N}$-glycan structures, a property, which is currently not achievable with any other system for the production of human-type $\mathrm{N}$-glycans. However, attention is required to ascertain that proteins are fully glycosylated and different means are available in order to increase $\mathrm{N}$ glycosylation efficiency. 
An aspect, which needs to be address in the future, is the effects of the tailored N-glycans on protein folding and cellular quality control systems. N-glycans play a critical role in the recognition of glycoproteins by the folding machinery, e.g. calnexin-calreticulin cycle. Furthermore, they act as signature for the recognition of missfolded or slowly-folding protein by Htm1 or Yos9 proteins, which are involved in ER associated protein degradation [1]. At the moment, no information exists how the engineering of the LLO biosynthesis affects these processes, however, it is obvious that in cells lacking Alg3 or Alg3 and Alg11 activities, these cellular quality control processes are bypassed as the $\mathrm{N}$-glycan determinants required for the recognition are missing. Therefore, studies are needed to investigate the effects of glycoengineering on protein quality. 


\section{Figure legends}

FIGURE 1. Overview of yeast and mammalian N-linked glycosylation pathway and strategies to generate humanized N-glycans in yeasts and fungi. A) Formation of the lipid-linked oligosaccharide (LLO) is initiated at the cytoplasmic side of the ER by Alg7. A Man ${ }_{5} \mathrm{GlcNAc}_{2}$ structure is assembled on the cytoplasmic side by the consecutive action of Alg13/14, Alg1, Alg2 and Alg11. This structure is flipped into the ER lumen, where modification of the LLO is initiated by Alg3. The B-branch is completed by the action of Alg9 after which the C-branch is started by Alg12 and completed by Alg9. The triglucosyl cap is added by Alg6, Alg8 and Alg10 generating a GlcNAc $_{3} \mathrm{Man}_{9} \mathrm{GlcNAc}_{2}$. This structure serves a substrate for the oligosaccharyltransferase complex (OST). After folding of the protein, the terminal mannose of the B-branch is removed. The assembly of LLO and the transfer by OST are conserved in yeast and mammals. Structural diversity of N-glycans is generated in the Golgi apparatus. In mammals, N-glycans of glycoproteins destined to the Golgi apparatus undergo trimming and transfer reactions. First Golgi localized $\alpha 1,2$ mannosidase (ManI) is trimming the $\alpha 1,2$ mannose residues, after which GnTI is adding the first GlcNAc residue. The remaining mannose residues are trimmed off by ManII, which create the substrate for GnTII that adds a second GlcNAc residue. A plethora of glycosyltransferases is present in the Golgi apparatus of mammals creating a highly diverse N-glycans. In contrast to mammals, yeast only performs mannosylation of the N-glycan in the Golgi-apparatus. By properly localizing the mammalian mannosidases and glycosyltransferases into the Golgi-apparatus of yeast, human-like N-glycans can be generated.

B) to E) Alternative pathways were designed to generate the substrate for GnTI and GnTII. By introducing a fungal $\alpha 1,2-$ mannosidase into the ER ( $\alpha 1,2 \mathrm{Man})$, ManI activity is not required in the Golgi and only GnTI, ManII and GnTII have to be expressed. C) Deletion of the ALG3 gene generates mainly a Man ${ }_{5} \mathrm{GlcNAc}_{2}$ structure, which can be transferred onto proteins. The ALG3 deletion leads to hypoglycosylation as the triglucosyl-cap of the A-branch, which is one of the key determinants for recognition by the OST, is missing. Only ManI, GnTI, and GnTII are required to generate a complex N-glycan. D) In this strategy the ALG3 deletion is combined with the overexpression of a ER-localized fungal $\alpha 1,2-m a n n o s i d a s e ~(\alpha 1,2 \mathrm{Man})$, which creates a $\mathrm{Man}_{3} \mathrm{GlcNAc}_{2}$. In order to attenuate hypoglycosylation, the ALG6 gene is overexpressed, which is adding the first glucose. E) Generation of a biosynthetic pathway to Man ${ }_{3} \mathrm{GlcNAc}_{2}$ structure. Deletion of $A L G 11$ and $A L G 3$ genes leads to the generation of $\mathrm{Man}_{3} \mathrm{GlcNAc}_{2}$ which serves as substrate for GnTI and GnTII. In order to compensate for hypoglycosylation, an artificial flippase 
Flc2* and a protozoan oligosaccharyltransferase (POT) have been introduced into the strain, leading to complete glycosylation of proteins.

Blue circles: glucose, green circles: mannose, blue squares: $\mathrm{N}$-acetylglucosamine, light blue circles: structure is not or only partially glucosylated.

FIGURE 2. Structure of eukaryotic dolichol-linked oligosaccharide. Linkages and the enzymes catalysing the respective steps are given. A, B and C denote the A-, B- and C-branch of the LLO. Deletion of $A L G 11$ gene prevents formation of the A-branch. Deletion of ALG3 gene prevents formation the B- and C-branch mainly generating a Man ${ }_{5} \mathrm{GlcNAc}_{2}$ structure. Glucosylation of the A-branch is strongly impaired in $\Delta a \lg 3$ mutant strains. Blue circles: glucose, green circles: mannose, blue squares: $\mathrm{N}$-acetylglucosamine 


\section{References}

[1] Helenius A, Aebi M. Roles of N-linked glycans in the endoplasmic reticulum. Annu Rev Biochem 2004;73:1019-49.

[2] Stanley P, Schachter H, Taniguchi N. N-Glycans. Essentials of Glycobiology, New York: Cold Spring Harbor Laboratory Press; 2009.

[3] Jefferis R. Recombinant antibody therapeutics: the impact of glycosylation on mechanisms of action. Trends Pharmacol Sci 2009;30:356-62.

[4] Ferrara C, Stuart F, Sondermann P, Brunker P, Umana P. The carbohydrate at FcgammaRIIIa Asn-162. An element required for high affinity binding to non-fucosylated IgG glycoforms. $\mathrm{J}$ Biol Chem 2006;281:5032-6.

[5] Sinclair AMM, Elliott S. Glycoengineering: the effect of glycosylation on the properties of therapeutic proteins. J Pharm Sci 2005;94:1626-35.

[6] Higgins E. Carbohydrate analysis throughout the development of a protein therapeutic. Glycoconj J 2010;27:211-25.

[7] Jacobs PP, Callewaert N. N-glycosylation engineering of biopharmaceutical expression systems. Curr Mol Med 2009;9:774-800.

[8] Wildt S, Gerngross TU. The humanization of N-glycosylation pathways in yeast. Nat Rev Microbiol 2005;3:119-28.

[9] Parsaie Nasab F, Aebi M, Bernhard G, Frey AD. A combined system for engineering glycosylation efficiency and glycan structure in Saccharomyces cerevisiae. Applied and Environmental Microbiology 2013;79:997-1007.

[10] Nett JH, Stadheim TA, Li H, Bobrowicz P, Hamilton SR, Davidson RC, et al. A combinatorial genetic library approach to target heterologous glycosylation enzymes to the endoplasmic reticulum or the Golgi apparatus of Pichia pastoris. Yeast 2011;28:237-52.

[11] Hamilton SRR, Bobrowicz P, Bobrowicz B, Davidson RCC, Li H, Mitchell T, et al. Production of complex human glycoproteins in yeast. Science 2003;301:1244-6.

[12] Narasimhan S, Stanley P, Schachter H. Control of glycoprotein synthesis. Lectin-resistant mutant containing only one of two distinct $\mathrm{N}$-acetylglucosaminyltransferase activities present in wild type Chinese hamster ovary cells. J Biol Chem 1977;252:3926-33.

[13] Aebi M, Gassenhuber J, Domdey H, te Heesen S. Cloning and characterization of the ALG3 gene of Saccharomyces cerevisiae. Glycobiology 1996;6:439-44.

[14] Vervecken W, Kaigorodov V, Callewaert N, Geysens S, De Vusser K, Contreras R. In vivo synthesis of mammalian-like, hybrid-type N-glycans in Pichia pastoris. Appl Environ Microbiol 2004;70:2639-46. 
[15] Helenius J, Ng DT, Marolda CL, Walter P, Valvano MA, Aebi M. Translocation of lipidlinked oligosaccharides across the ER membrane requires Rft1 protein. Nature 2002;415:447-50.

[16] Davidson RC, Nett JH, Renfer E, Li H, Stadheim TA, Miller BJ, et al. Functional analysis of the ALG3 gene encoding the Dol-P-Man: Man5GlcNAc2-PP-Dol mannosyltransferase enzyme of P. pastoris. Glycobiology 2004;14:399-407.

[17] De Pourcq K, Tiels P, Van Hecke A, Geysens S, Vervecken W, Callewaert N. Engineering Yarrowia lipolytica to produce glycoproteins homogeneously modified with the universal Man3GlcNAc2 N-glycan core. PloS One 2012;7:e39976.

[18] Cipollo JF, Trimble RB, Chi JH, Yan Q, Dean N. The yeast ALG11 gene specifies addition of the terminal alpha 1,2-Man to the Man5GlcNAc2-PP-dolichol N-glycosylation intermediate formed on the cytosolic side of the endoplasmic reticulum. The Journal of Biological Chemistry 2001;276:21828-40.

[19] Kelleher DJ, Gilmore R. An evolving view of the eukaryotic oligosaccharyltransferase. Glycobiology 2006;16:47R-62R.

[20] Samuelson J, Banerjee S, Magnelli P, Cui J, Kelleher DJ, Gilmore R, et al. The diversity of dolichol-linked precursors to Asn-linked glycans likely results from secondary loss of sets of glycosyltransferases. Proc Natl Acad Sci U S A 2005;102:1548-53.

[21] Izquierdo L, Schulz BL, Rodrigues JA, Guther ML, Procter JB, Barton GJ, et al. Distinct donor and acceptor specificities of Trypanosoma brucei oligosaccharyltransferases. Embo J 2009;28:2650-61.

[22] Hese K, Otto C, Routier FH, Lehle L. The yeast oligosaccharyltransferase complex can be replaced by STT3 from Leishmania major. Glycobiology 2009;19:160-71.

[23] Nasab FP, Schulz BL, Gamarro F, Parodi AJ, Aebi M. All in one: Leishmania major STT3 proteins substitute for the whole oligosaccharyltransferase complex in Saccharomyces cerevisiae. Mol Biol Cell 2008;19:3758-68.

[24] Choi BK, Warburton S, Lin H, Patel R, Boldogh I, Meehl M, et al. Improvement of N-glycan site occupancy of therapeutic glycoproteins produced in Pichia pastoris. Appl Microbiol Biotechnol 2012. 
A)

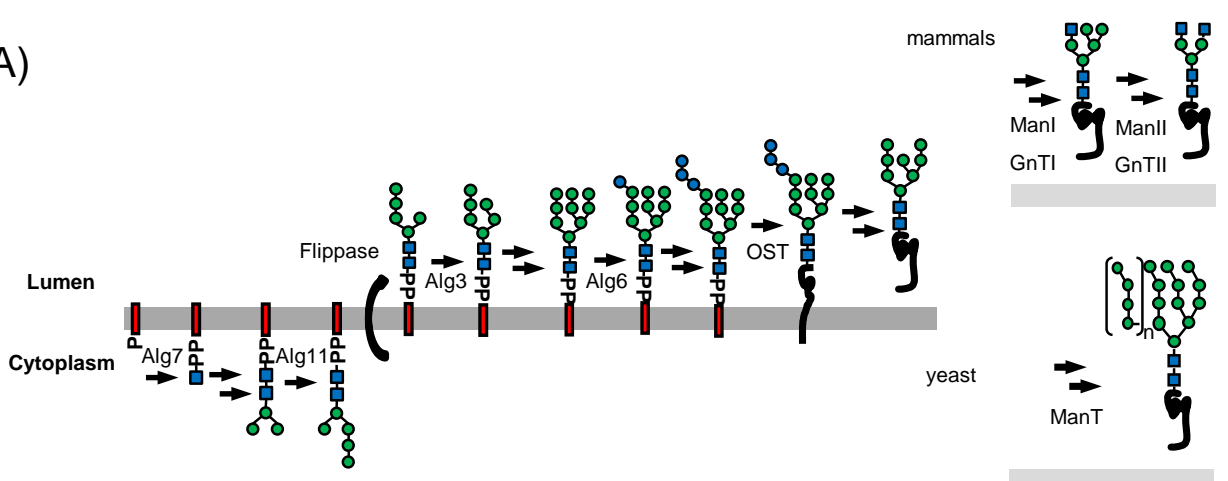

B)

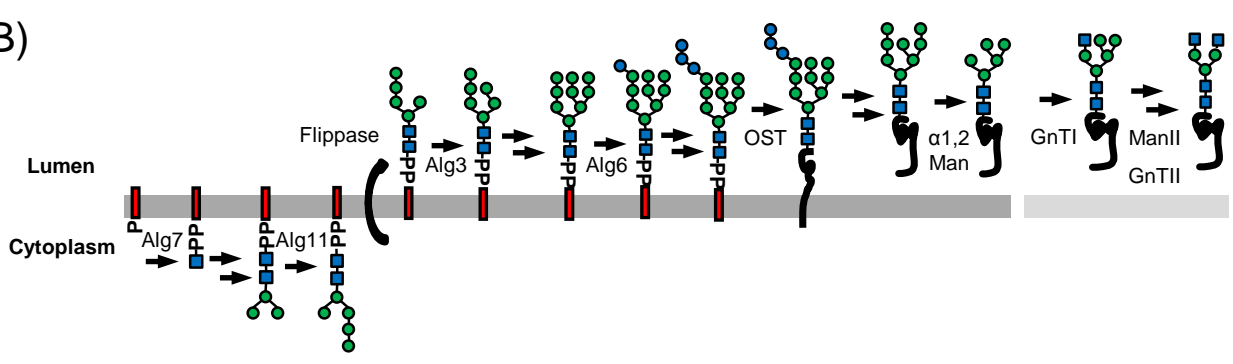

C)
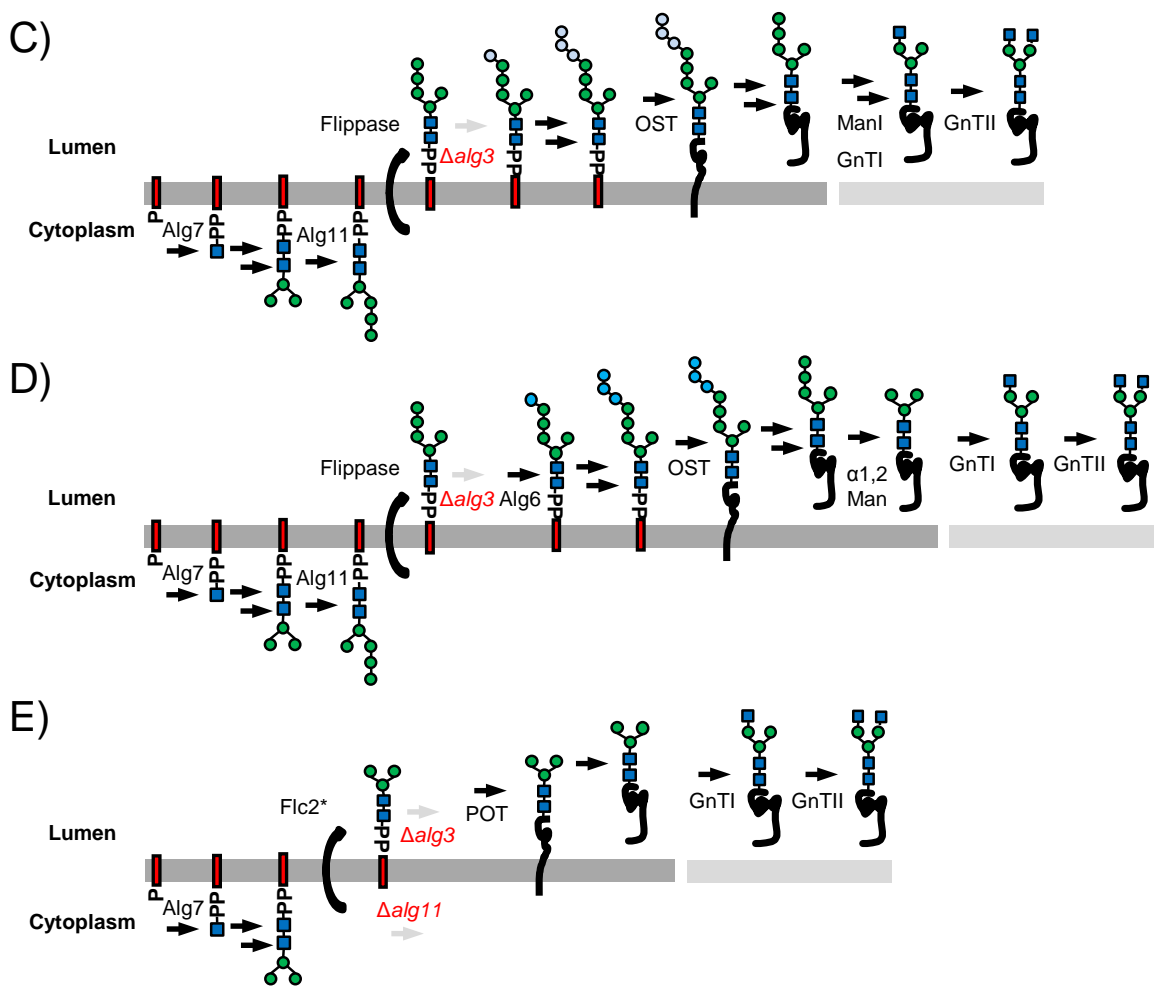


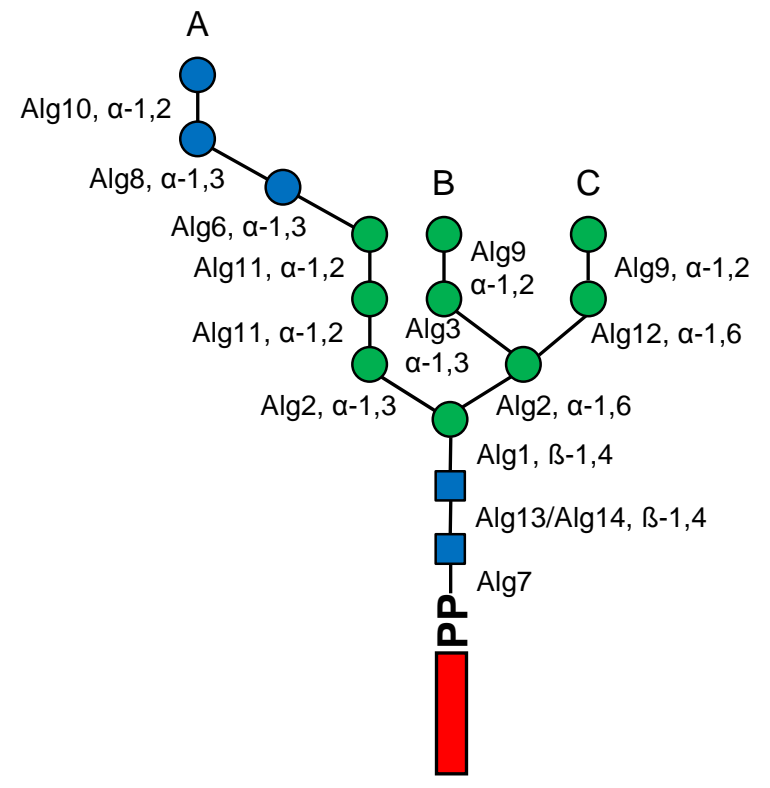

